\title{
Ames hypopituitary dwarf mice demonstrate imbalanced myelopoiesis between bone marrow and spleen
}

\author{
Maegan L. Capitano ${ }^{1}$, Brahmananda R. Chitteti ${ }^{2}$, Scott Cooper ${ }^{1}$, Edward F. Srour ${ }^{2}$, Andrzej \\ Bartke $^{3}$, and Hal E. Broxmeyer ${ }^{1}$ \\ ${ }^{1}$ Department of Microbiology and Immunology, Indiana University School of Medicine, \\ Indianapolis, Indiana, USA \\ ${ }^{2}$ Department of Medicine, Indiana University School of Medicine, Indianapolis, Indiana, USA \\ ${ }^{3}$ Department of Internal Medicine, Geriatrics Research, Southern Illinois University School of \\ Medicine, Springfield, Illinois, USA
}

\section{Abstract}

Ames hypopituitary dwarf mice are deficient in growth hormone, thyroid-stimulating hormone, and prolactin. The phenotype of these mice demonstrates irregularities in the immune system with skewing of the normal cytokine milieu towards a more anti-inflammatory environment. However, the hematopoietic stem and progenitor cell composition of the bone marrow (BM) and spleen in Ames dwarf mice has not been well characterized. We found that there was a significant decrease in overall cell count when comparing the BM and spleen of 4-5 month old dwarf mice to their littermate controls. Upon adjusting counts to differences in body weight between the dwarf and control mice, the number of granulocyte-macrophage progenitors, confirmed by immunophenotyping and colony-formation assay was increased in the BM. In contrast, the numbers of all myeloid progenitor populations in the spleen were greatly reduced, as confirmed by colony-formation assays. This suggests that there is a shift of myelopoiesis from the spleen to the $\mathrm{BM}$ of Ames dwarf mice; however, this shift does not appear to involve erythropoiesis. The reasons for this unusual shift in spleen to marrow hematopoiesis in Ames dwarf mice are yet to be determined but may relate to the decreased hormone levels in these mice.

\section{Keywords}

Ames dwarf mice; hematopoietic progenitor cell; growth hormone; prolactin; thyroid-stimulating hormone

(C) 2015 Published by Elsevier Inc.

Corresponding Author: Hal E. Broxmeyer, Ph.D., Department of Microbiology and Immunology, Indiana University School of Medicine, R2 Room 302, 950 West Walnut Street, Indianapolis, Indiana 46202, hbroxmey @iupui.edu, Phone Number: (317) 274-7510, Fax Number: (317) 274-7592.

Publisher's Disclaimer: This is a PDF file of an unedited manuscript that has been accepted for publication. As a service to our customers we are providing this early version of the manuscript. The manuscript will undergo copyediting, typesetting, and review of the resulting proof before it is published in its final citable form. Please note that during the production process errors may be discovered which could affect the content, and all legal disclaimers that apply to the journal pertain. 


\section{Introduction}

The pituitary gland secretes several hormones including: growth hormone $(\mathrm{GH})$, prolactin (PRL) and thyroid-stimulating hormone (TSH). These hormones are secreted by three specific adenohypophyseal cell types: samatotrophs which produce $\mathrm{GH}$ in response to $\mathrm{GH}-$ releasing hormone, lactotrophs which produce PRL in response to estrogen, progesterone, and thyrotropin-releasing hormone, and thyrotrophs which produce TSH in response to thyrotropin-releasing hormone. These hormones are essential for growth, maintaining blood pressure, some aspects of pregnancy, child birth, and nursing, kidney function, pain relief, regulating body temperature and the function of sex organs and thyroid glands. The Ames dwarf mice are used to study the role of hypopituitarism on various biological functions. Ames dwarf mice are homozygous for a recessive loss of function mutation at the Prop1 locus (Prop1 ${ }^{\mathrm{df} / \mathrm{df}}$ ). ${ }^{[1-3]}$ The loss of function mutation at Prop1 interferes with development of the adenohypophyseal cell types, thus leading to deficiencies in GH, PRL and TSH. These hormone deficiencies also lead to suppressed circulating levels of insulin-like growth factor 1 (IGF-1), thyroid hormones (e.g. thyroxin and triiodothyronine), insulin and glucose ${ }^{[1,4]}$ Therefore, Ames dwarf mice demonstrate increased insulin sensitivity, glucose tolerance and hypothyroidism.

This significant alteration to the physiology of hypopituitary mice leads to a very unique phenotype. First, Prop $1^{\mathrm{df} / \mathrm{df}}$ mice are dwarfs that demonstrate a significantly longer lifespan than Prop $1^{+/+}$or Prop $1^{+/ d f}$ littermates with an $\sim 50 \%$ longer lifespan in males and a $>60 \%$ longer lifespan in females. ${ }^{[4,5]}$ Second, they have delayed occurrences of fatal neoplastic diseases suggesting that these mice do age like normal mice, but differences in the aging phenotype do not occur until the mice are much older. ${ }^{[5,6]}$ Finally, hypopituitary mice demonstrate a significant reduction in the number of bone marrow cells, splenocytes and thymocytes even after adjusting for differences in body weight between dwarf mice and their littermate controls. ${ }^{[7-12]}$

B cell development in the bone marrow of $\sim 1-4$ month old hypopituitary mice is defective as indicated by a decrease in pre-, pro- and total B cell numbers when compared to littermate controls. ${ }^{[9,12,13]} \mathrm{T}$ cell development is also deficient in these mice as indicated by a reduction in double positive $\left(\mathrm{CD} 4^{+} \mathrm{CD} 8^{+}\right) \mathrm{T}$ cells in the thymus and the abnormal presence of double positive $\mathrm{T}$ cells in the lymph nodes. ${ }^{[9,12,13]} \mathrm{In}$ addition to abnormal $\mathrm{T}$ and $\mathrm{B}$ cell development, functional studies have shown that both cell-mediated and humoral immunity is compromised in hypopituitary mice. ${ }^{[7,10,11]}$ Ames dwarf mice have an overall reduction in the ability of $\mathrm{T}$ cells to respond to super antigen compared to their littermate controls.

Ectopic pituitary transplants restored Ames dwarf mice to immunocompetence by enhancing the number of lymphocytes and their natural killer activity suggesting that restoring PRL levels alters the immunodeficiency seen in Ames dwarf mice. ${ }^{[14]}$ These findings are also observed in other pituitary dwarf syndromes such as the Snell-Bagg/Snell dwarf mice and in mice and humans in which GH, PRL, thyroxine, and IGF-1 functions were impaired.$^{[8,11,13-15]}$ However, it is important to note that in the Snell-Bagg dwarf mice, the differences seen in the immune system are dependent on when the mice were weaned. ${ }^{[16]}$ 
A microarray analysis of 34,000 genes in the peripheral blood leukocytes from 7 month old Ames dwarf mice identified 6 main genes which had altered expression in Ames dwarf mice compared to littermate controls: casp3, bcl2, IL4, mapk14, TGF $\beta 1$ and pcrk. ${ }^{[17]}$ The function of these genes suggests that Ames dwarf mice may have functional changes in apoptosis, B and T cell homeostasis, prostaglandin synthesis, humoral immunity, chemokine activity, complement activation, and wound healing suggesting that Ames dwarf mice have several anti-inflammatory pathways activated. This is further supported by the fact that Ames dwarf mice have increased levels of adiponectin that can act as an anti-inflammatory factor. ${ }^{[18-20]} \mathrm{GH}$ regulates the level of adiponectin produced by adipose tissues and in the absence of GH signaling adipose tissue increases its release of adiponectin and reduces the secretion of pro-inflammatory cytokines such as tumor necrosis factor alpha (TNFa) and interleukin (IL)-6. ${ }^{[21]}$ Interestingly, disruption of GH receptor in adipose tissue alone results in normal adiponectin levels unlike what occurs in global GH receptor knockout mice suggesting that GH indirectly regulates adiponectin levels. ${ }^{[22]}$

Although it has been fairly well established that pituitary hormonal deficiencies lead to abnormalities in the immune response, little is known of what is occurring to the hematopoietic stem cell (HSC) and the hematopoietic progenitor cell (HPC) populations in the bone marrow and spleen of hypopituitary mice. It has been previously published that there was an increase in a $\mathrm{Lin}^{-} \mathrm{Sca}{ }^{+} \mathrm{CD}_{4} 5^{+}$population and a trend increase in clonogeneic progenitor cells in the bone marrow of Ames dwarf mice when compared to littermate controls. ${ }^{[23]}$ In this paper, through the utilization of a more extensive panel of markers for immunophenotyping and by colony-formation assays, we examined HSC and HPC composition of the bone marrow and spleen in greater detail to elucidate alterations in hypopituitary Ames dwarf mice.

\section{Materials and Methods}

\section{Mice}

Non-obese diabetic (NOD)/ severe combined immunodeficiency (SCID) mice (8-10 week old females) were obtained from an on-site breeding core facility at Indiana University School of Medicine. Male and female Ames dwarf (Prop1 ${ }^{\mathrm{df}}$ ) homozygous (df/df) and littermate control (+/df) mice were bred at Southern Illinois University then transferred to Indiana University School of Medicine. Details of the animal husbandry were described previously. ${ }^{[24]}$ Briefly, Ames df/df mice were produced by mating heterozygous females and homozygous mutant males. All breeding protocols were approved by the Southern Illinois University Laboratory Animal Care Committee. All further animal procedures were approved by the Indiana University Committee on Use and Care of Animals. Animals were maintained under temperature-and light-controlled conditions $\left(21-24^{\circ} \mathrm{C}, 12\right.$ hour light/12 hour dark cycle). Animals were group-housed according to age, sex and genotype. Mice were fed ad libitum. Mice were between 4-5 months of age at time of use. Immediately following euthanization, mice were weighed utilizing a Mettler PM2000 scale (Mettler Toledo; Columbus, $\mathrm{OH}$ ) and femurs and spleens excised. 


\section{Flow Cytometry}

Immunophenotyping of stem and progenitor cells was performed by flushing femurs of Ames dwarf (df/df) or littermate control (+/df) mice and incubating the bone marrow cells with fluorochrome conjugated anti-mouse antibodies in PBS at room temperature for 25 minutes. One $\mu \mathrm{g}$ antibody was used per one million cells for each antibody. All antibodies were purchased from either BD Biosciences (San Diego, CA) or BioLegend (San Diego, $\mathrm{CA}$ ). For bone marrow HSC and HPC analysis the following panel of antibodies were used: FITC-conjugated anti-mouse lineage cocktail (anti-CD3/Gr-1/CD11b/B220/Ter-119; BioLegend), PE-Cy7-conjugated anti-mouse Sca1 (clone D7), APC-H7-conjugated antimouse c-Kit (clone 2B8), PE-conjugated anti-mouse CD34 (clone RAM34), APCconjugated anti-mouse Flk2 (clone A2F10.1), PerCp-Cy5.5-conjugated anti-mouse Fc $\gamma \mathrm{R}$ (clone 2.4G2) and BV421-conjugated anti-mouse IL-7R (clone SB/199) all purchased from BD Biosciences with their appropriate isotype controls. Long term-hematopoietic stem cells (LT-HSC) were defined as $\mathrm{Lin}^{-} \mathrm{Sca}^{+}{ }^{+} \mathrm{c}-\mathrm{Kit}^{+} \mathrm{CD} 34^{-} \mathrm{Flk} 2^{-}$. Short term (ST)-HSCs are defined as $\mathrm{Lin}^{-} \mathrm{Sca}{ }^{+} \mathrm{C}-\mathrm{Kit}^{+} \mathrm{CD} 34^{+} \mathrm{Flk2} 2^{-}$. Multipotent progenitors (MPP) were defined as $\mathrm{Lin}^{-} \mathrm{Sca}{ }^{+} \mathrm{c}-\mathrm{Kit}^{+} \mathrm{CD} 34^{+} \mathrm{Flk2}{ }^{+}$. Common myeloid progenitors (CMP) were defined as $\mathrm{Lin}^{-} \mathrm{Sca}{ }^{-} \mathrm{c}-\mathrm{Kit}^{+} \mathrm{CD} 34^{\mathrm{int}} \mathrm{Fc} \gamma \mathrm{R}^{\mathrm{lo}}$. Granulocyte-macrophage progenitors (GMP) were defined as $\mathrm{Lin}^{-} \mathrm{Sca}^{-} \mathrm{c}-\mathrm{Kit}^{+} \mathrm{CD} 34^{\mathrm{hi}} \mathrm{Fc} \gamma \mathrm{R}^{\mathrm{hi}}$. Megakaryocyte-erythrocyte progenitors (MEP) were defined as $\mathrm{Lin}^{-} \mathrm{Sca}{ }^{-} \mathrm{c}-\mathrm{Kit}^{+} \mathrm{CD} 34^{\mathrm{lo}} \mathrm{Fc} \gamma \mathrm{R}^{\mathrm{lo}}$. Common lymphoid progenitors (CLP) were defined as $\mathrm{Lin}^{-} \mathrm{Sca} 1{ }^{\mathrm{lo}} \mathrm{c}-\mathrm{Kit}^{\mathrm{lo}} \mathrm{Flk} 2^{+} \mathrm{IL}-7 \mathrm{R}^{+}$. Data were acquired on an LSRII flow cytometer (BD Biosciences). Single color compensation and isotype controls were included in each experiment. Data analysis was performed using FlowJo 7.6.3 software (TreeStar, WA). Gates were determined using fluorescence minus-one controls. An example of the gating strategy to determine the percent of each population is given in Supplementary Figure 1. The percent of each population was used to calculate the absolute number of each phenotype of stem and progenitor cells per femur. Once the number of cells per femur was determined the number of cells for the dwarf mouse was adjusted based on the difference in weight using the following formula: [(average weight of littermate control mice/individual dwarf mouse weight)* the number of cells per femur].

\section{HPC assays}

For HPC assays performed in agar, bone marrow cells flushed from the femurs of Ames dwarf (df/df) or littermate control (+/df) mice were plated at $5 \times 10^{4}$ cells $/ \mathrm{mL}$ in $0.3 \%$ semisolid agar medium with 10\% FBS (Fisher Scientific; Watham, MA) that did or did not contain $10 \mathrm{ng} / \mathrm{mL}$ recombinant mouse granulocyte macrophage colony-stimulating factor (rmGM-CSF; R\&D Systems; Minneapolis, MN), 10ng/mL recombinant mouse macrophage colony-stimulating factor (rmM-CSF; R\&D Systems), $10 \mathrm{ng} / \mathrm{mL}$ recombinant mouse interleukin-3 (rmIL-3; R\&D Systems) and $50 \mathrm{ng} / \mathrm{mL}$ recombinant mouse stem cell factor (rmSCF; R\&D Systems). Colonies were scored after 6 days of incubation at $5 \% \mathrm{CO}_{2}$ and lowered (5\%) $\mathrm{O}_{2}$ in a humidified chamber. For HPC assays performed in methylcellulose, bone marrow cells flushed from femurs or splenocytes isolated from spleens of Ames dwarf $(\mathrm{df} / \mathrm{df})$ or littermate control (+/df) mice were plated at $2.5 \times 10^{4}$ (spleen) or $5 \times 10^{4}$ (bone marrow) cells $/ \mathrm{mL}$ in $1 \%$ methylcellulose culture medium with $0.1 \mathrm{mM}$ hemin (SigmaAldrich; St. Louis, MO), 30\% FBS, 1U/mL recombinant human erythropoietin (rhuEPO; R\&D Systems), 50ng/mL rmSCF (R\&D Systems), and 5\% vol/vol pokeweed mitogen 
mouse spleen cell conditioned medium. Colonies were scored after 6 days of incubation at $5 \% \mathrm{CO}_{2}$ and lowered (5\%) $\mathrm{O}_{2}$ in a humidified chamber, and granulocyte-macrophage colony-forming units (CFU-GM), erythrocyte burst-forming unit (BFU-E), and granulocyte, erythrocyte, monocyte, megakaryocyte CFU (CFU-GEMM) progenitors were distinguished by examining the morphology of the colonies. For both the agar and methylcellulose assays, total number of colonies per femur or spleen was calculated. Once the number of colonies per femur/spleen was determined, the number of colonies for the dwarf mouse was adjusted based on the difference in weight of the Ames dwarf.

\section{Short-term engraftment studies}

Recipient NOD/SCID mice received a single dose of 3.5Gy of total body irradiation (TBI, ${ }^{137} \mathrm{Cs}$ source) followed 24 hours later with an i.v. injection of $1 \times 10^{5}$ bone marrow cells isolated either from the femurs of Ames dwarf (df/df) or littermate control (+/df) mice. Peripheral blood was collected from the tail vein into heparinized microcapillary tubes (Fisher Scientific; Pittsburg, PA, USA) 1 and 2 months post transplantation. Following lysis of red blood cells using lysis buffer $\left(0.155 \mathrm{M} \mathrm{NH}_{4} \mathrm{Cl}, 0.01 \mathrm{M} \mathrm{KHCO}_{3}, 0.1 \mathrm{mM}\right.$ EDTA in $\mathrm{H}_{2} \mathrm{O}$; Sigma-Aldrich), cells were washed, blocked with both human and mouse gamma globulin, stained with PE-conjugated anti-mouse $\mathrm{H}-2 \mathrm{~K}^{\mathrm{k}}$, and fixed prior to flow analysis on a FACSCalibur utilizing BD CellQuest ${ }^{\mathrm{TM}}$ Pro software to determine percent $\mathrm{H}-2 \mathrm{~K}^{\mathrm{k}+}$ cells. This was done to determine the percentage of cells that were Ames in origin since Ames mice are $\mathrm{H}-2 \mathrm{~K}^{\mathrm{k}+}$ and NOD/SCID mice are not. Three female littermate controls (+/df) and dwarf (df/df) mice were used for these experiments. The bone marrow from each mouse was transplanted into 5 recipients. Therefore, there was a total of 15 NOD/SCID mice each that received either $+/ \mathrm{df}$ bone marrow or $\mathrm{df} / \mathrm{df}$ bone marrow.

\section{Statistical analysis}

For the body weight, cells counts, flow cytometry experiments and colony assays all data are the mean \pm SEM of 9 individual mice per group from 3 separate experiments. For Ames bone marrow engraftment studies, the average percent $\mathrm{H}-2 \mathrm{~K}^{\mathrm{k}+}$ cells of 15 individual mice per group was calculated \pm SEM. Student's two-tailed $t$ test was used to compare Ames dwarf mice to their littermate controls. $\mathrm{P}<0.05$ was considered significant.

\section{Results}

As has been previously published utilizing multiple different hypopituitary dwarf mouse models ${ }^{[7-12]}$, Ames dwarf mice demonstrate a significant reduction in body weight (2.47 fold less) and in the number of bone marrow cells ( 2.3 fold less) and splenocytes ( 4.16 fold less) when compared to littermate controls (Figure 1). The reduction in nucleated cells in the bone marrow correlates well with the overall difference in body weight between the two groups of mice; however, there is a greater reduction in the number of cells in the spleen then can be explained by the difference in weight (Figure 1D) suggesting that there is an abnormality that is occurring in the spleen of Ames dwarf mice either in the recruitment or maintenance of cell numbers within this organ. 
Ames dwarf mice are known to have abnormalities in both B and $\mathrm{T}$ cell development demonstrated by lower numbers of pre-, pro- and total B cells in the bone marrow and a reduction of double positive $T$ cells in the thymus. ${ }^{[9,12]}$ However, whether these changes in $\mathrm{T}$ and $\mathrm{B}$ cell numbers were due to changes in HSC and HPC numbers in the bone marrow and spleen has not been fully investigated. First we examined HSC and HPC population changes in the bone marrow via immunophenotyping. Bone marrow cells from the femurs of Ames dwarf and littermate control mice were stained with antibodies against lineage markers, Sca1, c-Kit, CD34, Flk2, Fc $\gamma$ R, and IL-7R in order to examine the long-term (LT)HSC, short-term (ST)-HSC, multipotent progenitor (MPP), common myeloid progenitor (CMP), granulocyte-macrophage progenitor (GMP), megakaryocyte-erythrocyte progenitor (MEP), and common lymphoid progenitor (CLP) populations by flow cytometry. When examining the percent population of HSCs and HPCs in the bone marrow, we found significant increases in the percent CMP and GMP populations but no change in the LTHSC, MPP, CLP, or MEP populations (Figure 2A). We also saw a decrease in the percent ST-HSC population. However, when we calculated the overall number of HSCs and HPCs and then adjusted cell numbers to the difference in body weight between the Ames dwarf mice and littermate controls, we saw no change in the number of ST-HSC and a very small, but significant, increase in LT-HSC (1.27 fold increase; Figure 2B). Most interestingly, we continued to see a significant increase in CMP (1.53 fold) and GMP (1.71 fold) suggesting that hematopoiesis in the bone marrow of Ames dwarf mice is skewed towards myelopoiesis. There was no significant difference in the CLP numbers suggesting that the difference in B and T cell development seen in Ames dwarf mice is most likely a later event in the maturation process of these cell populations.

To further characterize the progenitor populations in the bone marrow, colony-forming assays were utilized. First, bone marrow cells were placed in a semi-solid agar medium with or without GM-CSF, IL-3, M-CSF and SCF. The number of CFU-GM colonies per femur was calculated then adjusted for the difference in body weight between the Ames dwarf mice and littermate controls. There was a significant increase in the number of CFU-GM colonies following culture in IL-3 (2.36 fold increase) and M-CSF ( 2.52 fold increase) alone but not with GM-CSF alone when comparing Ames dwarf mice to littermate controls (Figure 3A). With the addition of SCF to the GM-CSF culture, which allows one to detect a more immature HPC population than that seen with only a colony-stimulating factor, we saw a significant increase in the number of CFU-GM colonies (1.48 fold increase) when comparing Ames dwarf mice to littermate controls. This is also true when combining IL-3 with SCF (2.09 fold increase) thus there are significant increases in both immature and mature subsets of CFU-GM. Next, bone marrow cells were placed in methylcellulose culture medium in the presence of hemin, EPO, SCF, and pokeweed mitogen spleen cell conditioned media which allows one to detect erythroid (BFU-E) and multipotent (CFUGEMM) progenitors. The number of colonies per femur was calculated then adjusted for the difference in body weight between the Ames dwarf mice and littermate controls. While, there was a trend towards decrease in numbers of erythroid (1.86 fold decrease) and multipotent ( 2.23 fold decrease) progenitors in the Ames dwarf mice the differences were not significant (Figure 3B). 
To characterize HPC numbers in the spleen, splenocytes were placed in methylcellulose culture medium in the presence of hemin, $\mathrm{EPO}, \mathrm{SCF}$, and pokeweed mitogen spleen cell conditioned media. Numbers of colonies per spleen were calculated then adjusted for differences in body weight between the Ames dwarf mice and littermate controls. In contrast to bone marrow, there was a significant 7.56 fold decrease in CFU-GM, 1.93 fold decrease in BFU-E, and 3.49 fold decrease in CFU-GEMM in the Ames dwarf mice compared to littermate controls (Figure 3C) suggesting that the overall number of progenitor cells in the spleens of dwarf mice is greatly reduced. This correlated well with the reduced overall number of splenocytes seen in dwarf mice when compared to littermate controls. These findings suggest that the Ames dwarf mice may be shifting the location of some of their hematopoietic function from the spleen (in which we see an overall decrease in HPC numbers) to the bone marrow (in which we see an increase in immature and mature subsets of granulocyte macrophage progenitor numbers).

Finally, we assessed the short-term engraftment capability of the bone marrow of Ames dwarf mice in sublethally irradiated NOD/SCID mice. NOD/SCID mice were utilized as host animals due to the mixed background of the Ames dwarf mice used in the present study. Ames mice $\left(\mathrm{H}-2 \mathrm{~K}^{\mathrm{k}+}\right)$ have a different haplotype then NOD/SCID mice $\left(\mathrm{H}-2 \mathrm{~K}^{\mathrm{k}-}\right)$. Therefore, to determine the number of blood cells that originated from Ames mice 1 and 2 months after transplantation, we used antibodies against $\mathrm{H}-2 \mathrm{~K}^{\mathrm{k}}$ and examined the blood by flow cytometry. We detected no difference in the short-term engraftment capabilities of Ames dwarf versus littermate control bone marrow (Figure 4). However, since NOD/SCID mice have normal pituitaries, hormone levels in these mice might negate any phenotype we may have seen in the GH, PRL, and TSH-deficient Ames dwarf mice.

\section{Discusion}

Despite considerable knowledge gleamed on the role of hypopituitarism (where there are multiple neuroendocrine abnormalities) on the peripheral immune system, little is known about how hypopituitarism affects hematopoietic stem and progenitor cells. In this paper we utilized the Ames dwarf mice to examine the role of hypopituitarism on the hematopoietic stem and progenitor cell composition in the bone marrow and spleen. In mice, the spleen, as well as bone marrow, is an active hematopoietic organ. In these animals that lack GH, PRL, and TSH, we observed a drop in extramedullary hematopoiesis in the spleen as seen by a significant decrease in HPC numbers (CFU-GM, BFU-E and CFU-GEMM) as assessed by functional colony forming assays (Figure 3C). This correlated with a significant drop in cell count in the spleen even when taking into account the difference in body weight between the Ames dwarf mice and their littermate controls (Figure 1). In contrast, we saw an increase in phenotyped CMP and GMP numbers and CFU-GM, as determined by colony-formation assays, in the bone marrow (Figure 2 and Figure 3A). Thus, there is an apparant imbalance in myelopoiesis between the bone marrow and the spleen in the Ames dwarf mice.

Extramedullary hematopoiesis, the formation and development of hematopoietic cells outside the bone marrow, occurs in the spleen of mice throughout adulthood. ${ }^{[25-27]}$ Many have studied mechanisms by which this process is induced or upregulated (e.g. following bone marrow failure, myelostimulation, tissue inflammation, injury, and repair, or due to 
abnormal systemic or local chemokine production). ${ }^{[25,26]}$ Very little is known as to why there would be an imbalance in overall hematopoiesis with a decrease in extramedullary hematopoiesis in the spleen and an increase hematopoiesis in the bone marrow. It is possible that the abnormal systemic immune environment of the hypopituitary dwarf mice does not favor extramedullary hematopoiesis. GH regulates the levels of adiponectin (an adipocytederived hormone) which is needed to regulate several important metabolic processes such as blood glucose and fatty acid levels. ${ }^{[18-21]}$ In the absence of GH, adipose tissue increases its secretion of adiponectin thus modulating NF- $\kappa \mathrm{B}$-mediated signals in endothelial cells resulting in the inability of monocytes to properly home into tissue ${ }^{[28,29]}$ and by decreasing the production of pro-inflammatory cytokines (e.g. TNFa and IL-6) by adipose tissue. ${ }^{[20]}$ Perhaps this systemic anti-inflammatory environment is unfavorable for extramedullary hematopoiesis. In addition to its anti-inflammatory effects, adiponectin also can affect myelopoiesis. Yokota and colleagues ${ }^{[30]}$ demonstrated that myeloid progenitors can be directly influenced by adiponectin when added in a colony-forming assay resulting in inhibition of colony formation. However, when adiponectin and bone marrow stromal cells are added to the colony-forming assay there is an increase in myeloid progenitor cells. Thus, it is possible that differences in the microenvironment between the spleen and bone marrow may result in opposite effects of adiponectin (i.e. adiponectin stimulates stroma from the bone marrow to produce factors that stimulate myelopoiesis but not the stroma in the spleen). These hypotheses require further testing in order to determine what, if any, role adiponectin or other factors may or may not be playing in regulating hematopoiesis in the bone marrow or spleen of Ames dwarf mice.

The absence of GH, PRL, and TSH can all contribute to the phenomenon we see in the spleen and bone marrow of Ames dwarf mice as well. For example, the depletion of PRL using an anti-PRL antibody resulted in a decrease in BFU-E colonies in a colony-formation assay in the presence of GM-CSF, IL-3 and erythropoietin but did not change CFU-GM and CFU-GEMM numbers. ${ }^{[31,32]}$ Perhaps loss of PRL is why we see the trend towards a decrease in the bone marrow and the significant decrease we see in BFU-E in the spleen. The role of GH is less clear. Multiple reports differ on whether or not GH or IGF-1 has any effect on hematopoiesis. In vitro and in vivo studies that utilized recombinant GH or IGF-1 treatment suggested that these factors can regulate the survival and expansion of hematopoietic stem and progenitor cells, even reversing the age- and irradiation-induced losses of these populations. ${ }^{[12,13,33-41]}$ However, mice lacking GH (and thus having a decreased circulating level IGF-1) demonstrate normal hematopoiesis in the bone marrow and the spleen. ${ }^{[2,43]}$ Thus, the effects of GH and IGF-1 alone are not sufficient to cause the phenotype that we see in the Ames dwarf mice. Mice deficient in TSH demonstrate normal myelopoiesis. ${ }^{[4]}$ Therefore, the unique phenotype of an increase in myelopoiesis in the bone marrow but a decrease in the spleen of Ames dwarf mice, which is the opposite of what one would expect in an inflammatory situation, may be due to a combination of neuroendocrine abnormalities.

\section{Conclusions}

Hypopituitary Ames dwarf mice, which lack GH, PRL, and TSH, demonstrate decreased myelopoiesis in the spleen but enhanced myelopoiesis in the bone marrow. This suggests an 
imbalance in myelopoiesis between the spleen and bone marrow that may be due to neuroendocrine abnormalities. More work is necessary to elucidate a role for the neuroendocrine system in shifts in the balance of myelopoiesis between the bone marrow and spleen of mice.

\section{Supplementary Material}

Refer to Web version on PubMed Central for supplementary material.

\section{Acknowledgments}

This work was supported by NIH RO1s (HL056416, HL67384, and HL112669) and P01 DK090948 to H. E. B. and AG019899 to A. B. M. L. C. was supported by T32 DK007519 to H. E. B.

\section{Abbreviations}

GH

PRL

TSH

IGF-1

TNFa

IL

HSC

HPC

NOD

SCID

LT-HSC

ST-HSC

MPP

CMP

GMP

MEP

CLP

rmGM-CSF

rmM-CSF

rmIL-3

rmSCF

rhuEPO growth hormone

prolactin

thyroid-stimulating hormone

insulin-like growth factor-1

tumor necrosis factor alpha

interleukin

hematopoietic stem cell

hematopoietic progenitor cell

non-obese diabetic

severe combined immunodeficiency

long term-hematopoietic stem cell

short term-hematopoietic stem cell

multipotent progenitors

common myeloid progenitors

granulocyte-macrophage progenitors

megakaryocyte-erythrocyte progenitors

common lymphoid progenitors

recombinant mouse granulocyte macrophage colony-stimulating factor

recombinant mouse macrophage colony-stimulating factor

recombinant mouse interleukin-3

recombinant mouse stem cell factor

recombinant human erythropoietin 
CFU-GM granulocyte-macrophage colony-forming units

BFU-E erythrocyte burst-forming unit

CFU-GEMM granulocyte, erythrocyte, monocyte, megakaryocyte CFU

\section{References}

1. Bartke A. Histology of the Anterior Hypophysis, Thyroid and Gonads of Two Types of Dwarf Mice. Anat Rec. 1964; 149:225-235. [PubMed: 14173795]

2. Bartke, A. The Molecular Genetics of Aging. Berlin: Springer-Verlag; 2000. Delayed Aging in Ames Dwarf Mice. Relationships to Endocrine Function and Body Size; p. 181-202.

3. Sornson MW, Wu W, Dasen JS, et al. Pituitary lineage determination by the Prophet of Pit-1 homeodomain factor defective in Ames dwarfism. Nature. 1996; 384(6607):327-333. [PubMed: 8934515]

4. Bartke A, Coschigano K, Kopchick J, et al. Genes that prolong life: relationships of growth hormone and growth to aging and life span. J Gerontol A Biol Sci Med Sci. 2001; 56(8):B340-349. [PubMed: 11487592]

5. Brown-Borg HM, Borg KE, Meliska CJ, Bartke A. Dwarf mice and the ageing process. Nature. 1996; 384(6604):33. [PubMed: 8900272]

6. Ikeno Y, Bronson RT, Hubbard GB, Lee S, Bartke A. Delayed occurrence of fatal neoplastic diseases in ames dwarf mice: correlation to extended longevity. J Gerontol A Biol Sci Med Sci. 2003; 58(4):291-296. [PubMed: 12663691]

7. Baroni CD, Fabris N, Bertoli G. Effects of hormones on development and function of lymphoid tissues. Synergistic action of thyroxin and somatotropic hormone in pituitary dwarf mice. Immunology. 1969; 17(2):303-314. [PubMed: 5804539]

8. Dumont F, Robert F, Bischoff P. T and B lymphocytes in pituitary dwarf Snell-Bagg mice. Immunology. 1979; 38(1):23-31. [PubMed: 315916]

9. Duquesnoy RJ. Immunodeficiency of the thymus-dependent system of the Ames dwarf mouse. J Immunol. 1972; 108(6):1578-1590. [PubMed: 4402311]

10. Fabris N, Pierpaoli W, Sorkin E. Hormones and the immunological capacity. IV. Restorative effects of developmental hormones or of lymphocytes on the immunodeficiency syndrome of the dwarf mouse. Clin Exp Immunol. 1971; 9(2):227-240. [PubMed: 4936805]

11. Masternak MM, Bartke A. Growth hormone, inflammation and aging. Pathobiol Aging Age Relat Dis. 2012; 2

12. Murphy WJ, Durum SK, Longo DL. Role of neuroendocrine hormones in murine T cell development. Growth hormone exerts thymopoietic effects in vivo. J Immunol. 1992; 149(12): 3851-3857. [PubMed: 1460277]

13. Montecino-Rodriguez E, Clark R, Johnson A, Collins L, Dorshkind K. Defective B cell development in Snell dwarf (dw/dw) mice can be corrected by thyroxine treatment. J Immunol. 1996; 157(8):3334-3340. [PubMed: 8871629]

14. Esquifino AI, Villanua MA, Szary A, Yau J, Bartke A. Ectopic pituitary transplants restore immunocompetence in Ames dwarf mice. Acta Endocrinol (Copenh). 1991; 125(1):67-72. [PubMed: 1872127]

15. Taguchi T, Takenouchi H, Matsui J, et al. Involvement of insulin-like growth factor-I and insulinlike growth factor binding proteins in pro-B-cell development. Exp Hematol. 2006; 34(4):508518. [PubMed: 16569597]

16. Cross RJ, Bryson JS, Roszman TL. Immunologic disparity in the hypopituitary dwarf mouse. J Immunol. 1992; 148(5):1347-1352. [PubMed: 1531667]

17. Dhahbi J, Li X, Tran T, Masternak MM, Bartke A. Circulating blood leukocyte gene expression profiles: effects of the Ames dwarf mutation on pathways related to immunity and inflammation. Exp Gerontol. 2007; 42(8):772-788. [PubMed: 17611063] 
18. Louis A, Bartke A, Masternak MM. Effects of growth hormone and thyroxine replacement therapy on insulin signaling in Ames dwarf mice. J Gerontol A Biol Sci Med Sci. 2010; 65(4):344-352. [PubMed: 20200088]

19. Masternak MM, Panici JA, Wang F, Wang Z, Spong A. The effects of growth hormone (GH) treatment on GH and insulin/IGF-1 signaling in long-lived Ames dwarf mice. J Gerontol A Biol Sci Med Sci. 2010; 65(1):24-30. [PubMed: 19906822]

20. Wang Z, Al-Regaiey KA, Masternak MM, Bartke A. Adipocytokines and lipid levels in Ames dwarf and calorie-restricted mice. J Gerontol A Biol Sci Med Sci. 2006; 61(4):323-331. [PubMed: 16611697]

21. Ouchi N, Walsh K. Adiponectin as an anti-inflammatory factor. Clin Chim Acta. 2007; 380(1-2): 24-30. [PubMed: 17343838]

22. List EO, Berryman DE, Funk K, et al. The role of GH in adipose tissue: lessons from adiposespecific GH receptor gene-disrupted mice. Mol Endocrinol. 2013; 27(3):524-535. [PubMed: 23349524]

23. Kucia M, Masternak M, Liu R, et al. The negative effect of prolonged somatotrophic/insulin signaling on an adult bone marrow-residing population of pluripotent very small embryonic-like stem cells (VSELs). Age (Dordr). 2013; 35(2):315-330. [PubMed: 22218782]

24. Masternak MM, Al-Regaiey K, Bonkowski MS, et al. Divergent effects of caloric restriction on gene expression in normal and long-lived mice. J Gerontol A Biol Sci Med Sci. 2004; 59(8):784788. [PubMed: 15345726]

25. Johns JL, Christopher MM. Extramedullary hematopoiesis: a new look at the underlying stem cell niche, theories of development, and occurrence in animals. Vet Pathol. 2012; 49(3):508-523. [PubMed: 22262354]

26. Kim CH. Homeostatic and pathogenic extramedullary hematopoiesis. J Blood Med. 2010; 1:13-19. [PubMed: 22282679]

27. Wolber FM, Leonard E, Michael S, Orschell-Traycoff CM, Yoder MC, Srour EF. Roles of spleen and liver in development of the murine hematopoietic system. Exp Hematol. 2002; 30(9):10101019. [PubMed: 12225792]

28. Ouchi N, Kihara S, Arita Y, et al. Novel modulator for endothelial adhesion molecules: adipocytederived plasma protein adiponectin. Circulation. 1999; 100(25):2473-2476. [PubMed: 10604883]

29. Ouchi N, Kihara S, Arita Y, et al. Adiponectin, an adipocyte-derived plasma protein, inhibits endothelial NF-kappaB signaling through a cAMP-dependent pathway. Circulation. 2000; 102(11):1296-1301. [PubMed: 10982546]

30. Yokota T, Meka CS, Kouro T, et al. Adiponectin, a fat cell product, influences the earliest lymphocyte precursors in bone marrow cultures by activation of the cyclooxygenase-prostaglandin pathway in stromal cells. J Immunol. 2003; 171(10):5091-5099. [PubMed: 14607907]

31. Bellone G, Astarita P, Artusio E, et al. Bone marrow stroma-derived prolactin is involved in basal and platelet-activating factor-stimulated in vitro erythropoiesis. Blood. 1997; 90(1):21-27. [PubMed: 9207433]

32. Sun R, Gault RA, Welniak LA, Tian ZG, Richards S, Murphy WJ. Immunologic and hematopoietic effects of recombinant human prolactin after syngeneic bone marrow transplantation in mice. Biol Blood Marrow Transplant. 2003; 9(7):426-434. [PubMed: 12869956]

33. Claustres M, Chatelain P, Sultan C. Insulin-like growth factor I stimulates human erythroid colony formation in vitro. J Clin Endocrinol Metab. 1987; 65(1):78-82. [PubMed: 3584401]

34. Correa PN, Axelrad AA. Production of erythropoietic bursts by progenitor cells from adult human peripheral blood in an improved serum-free medium: role of insulinlike growth factor 1 . Blood. 1991; 78(11):2823-2833. [PubMed: 1954373]

35. Jardieu P, Clark R, Mortensen D, Dorshkind K. In vivo administration of insulin-like growth factorI stimulates primary B lymphopoiesis and enhances lymphocyte recovery after bone marrow transplantation. J Immunol. 1994; 152(9):4320-4327. [PubMed: 8157955]

36. Merchav S, Lake M, Skottner A. Comparative studies of the granulopoietic enhancing effects of biosynthetic human insulin-like growth factors I and II. J Cell Physiol. 1993; 157(1):178-183. [PubMed: 8408236] 
37. Merchav S, Tatarsky I, Hochberg Z. Enhancement of erythropoiesis in vitro by human growth hormone is mediated by insulin-like growth factor I. Br J Haematol. 1988; 70(3):267-271. [PubMed: 2849979]

38. Merchav S, Tatarsky I, Hochberg Z. Enhancement of human granulopoiesis in vitro by biosynthetic insulin-like growth factor I/somatomedin C and human growth hormone. J Clin Invest. 1988; 81(3):791-797. [PubMed: 2963830]

39. Murphy WJ, Durum SK, Anver MR, Longo DL. Immunologic and hematologic effects of neuroendocrine hormones. Studies on DW/J dwarf mice. J Immunol. 1992; 148(12):3799-3805. [PubMed: 1602129]

40. Sanders M, Sorba S, Dainiak N. Insulin-like growth factors stimulate erythropoiesis in serumsubstituted umbilical cord blood cultures. Exp Hematol. 1993; 21(1):25-30. [PubMed: 8417957]

41. Shaheen, M.; Broxmeyer, HE. Hematopoietic Cytokines and Growth Factors. In: Broxmeyer, HE., editor. Cord Blood: Biology, Transplantation, Banking, and Regulation. Bethesda, MD: AABB Press; 2011. p. 35-73.

42. Sharma Y, Flurkey K, Astle CM, Harrison DE. Mice severely deficient in growth hormone have normal hematopoiesis. Exp Hematol. 2005; 33(7):776-783. [PubMed: 15963853]

43. Stewart MH, Gutierrez-Martinez P, Beerman I, et al. Growth hormone receptor signaling is dispensable for HSC function and aging. Blood. 2014; 124(20):3076-3080. [PubMed: 25274507]

44. Montecino-Rodriguez E, Clark RG, Powell-Braxton L, Dorshkind K. Primary B cell development is impaired in mice with defects of the pituitary/thyroid axis. J Immunol. 1997; 159(6):2712-2719. [PubMed: 9300691] 
A

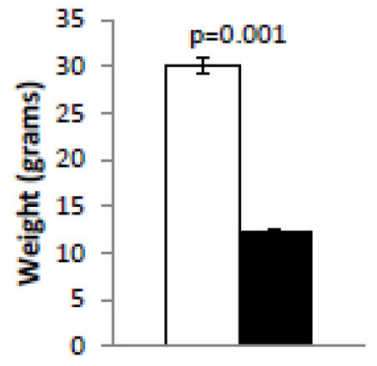

C

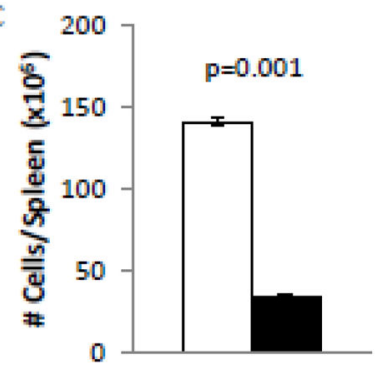

B

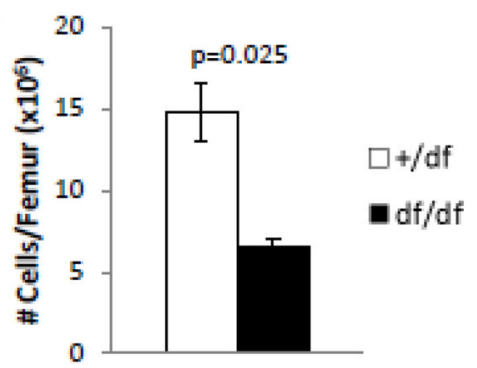

D

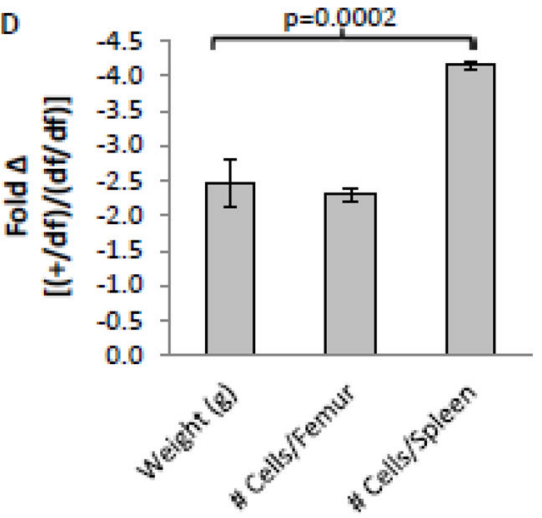

Figure 1.

Ames dwarf mice are smaller in weight with fewer nucleated cells in their femurs and spleens than littermate controls. (A) Ames dwarf (df/df) and littermate control (+/df) mice (4-5 months old) were weighed immediately prior to euthanasia. The femurs (B) and spleens (C) were collected from the animals, processed, and the number of nucleated cells counted. (D) The fold change of body weight and the number of cells in the bone marrow and spleen when comparing dwarf mice to their littermate controls are shown. Results (mean \pm SEM) are based on 9 individually assessed mice per group from 3 separate experiments. 
A
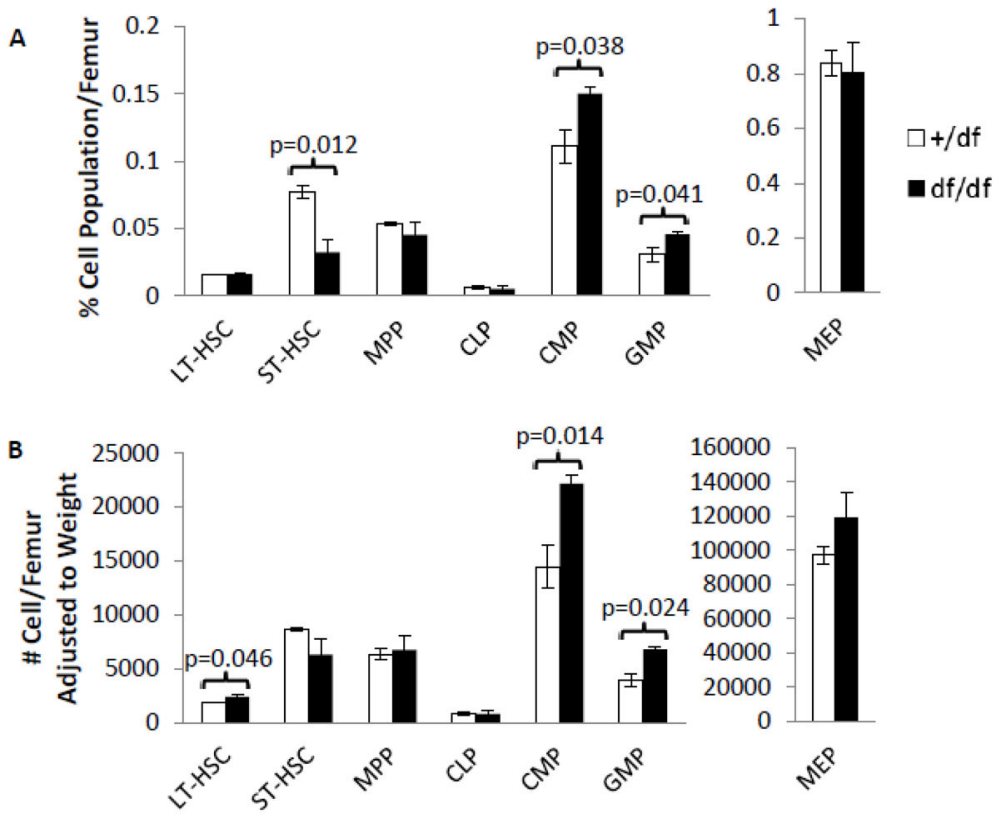

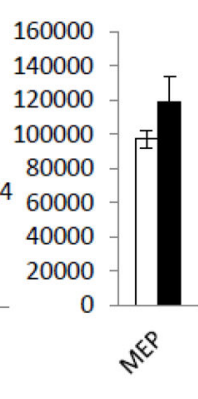

Figure 2.

The numbers of immunophenotyped LT-HSC, CMP, and GMP in the bone marrow are significantly higher in Ames dwarf (df/df) mice when compared to their littermate controls (+/df). The percent of each population examined (A) was used to calculate the absolute numbe of each phenotype of stem and progenitor cells (B). Results (mean \pm SEM) are based on 9 individually assessed mice per group from 3 separate experiments. 
A

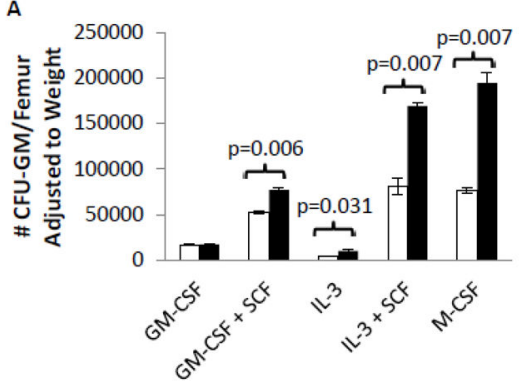

C

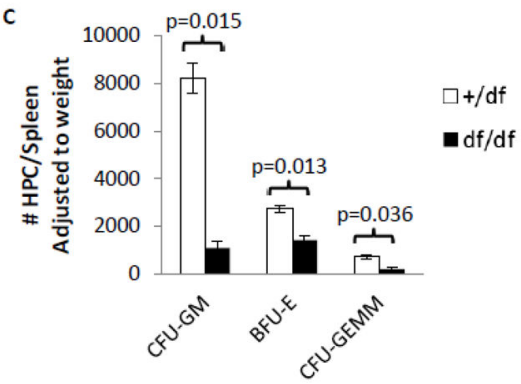

B

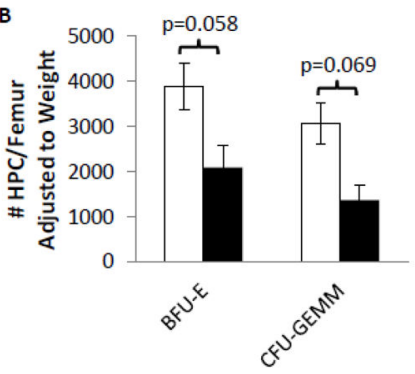

Figure 3.

The number of granulocyte-macrophage progenitor cells in the bone marrow of Ames dwarf mice is increased; however, an overall decrease in HPCs in the spleen is observed when compared to littermate controls. (A) Adjusted numbers of bone marrow CFU-GM from the femurs of Ames dwarf (df/df) and littermate control (+/df) mice. (B) Adjusted numbers of bone marrow BFU-E and CFU-GEMM from the femur of Ames dwarf (df/df) and littermate control (+/df) mice. (C) Adjusted numbers of hematopoietic progenitors in the spleen of Ames dwarf (df/df) and littermate control (+/df) mice. For all experiments results (mean \pm SEM) are based on 9 individually assessed mice per group from 3 separate experiments. 


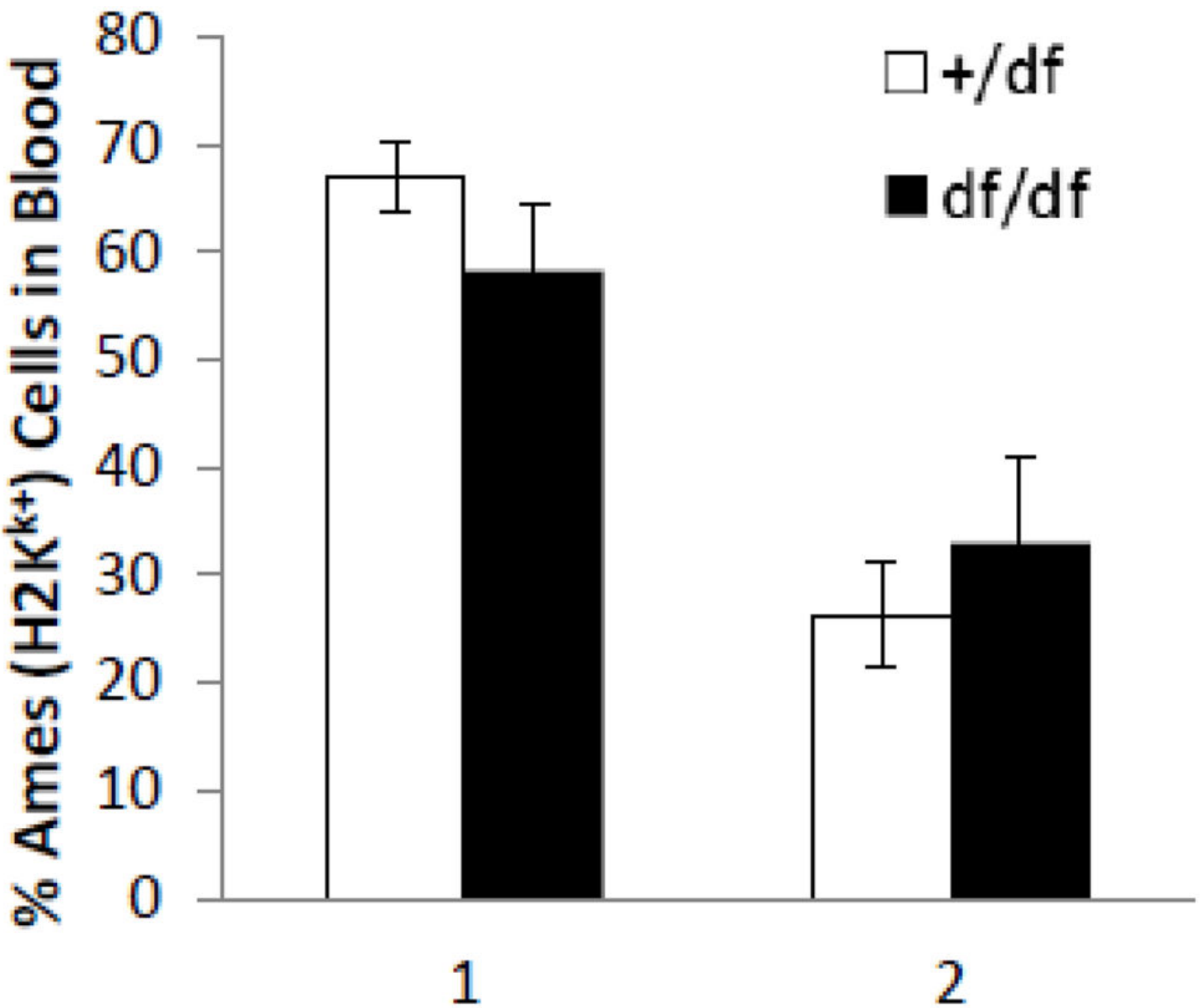

\section{Month Post Transplantation into NOD/SCID Mice}

Figure 4.

No significant difference in the short-term engraftment capability of bone marrow cells from Ames dwarf or littermate control mice. NOD/SCID mice received 3.5Gy total body irradiation followed one day later with $1 \times 10^{5}$ Ames dwarf (df/df) or littermate control (+/df) bone marrow cells. $\mathrm{H}-2 \mathrm{~K}^{\mathrm{k}}$ was used as a marker to determine whether cells originated from Ames $\left(\mathrm{H}-2 \mathrm{~K}^{\mathrm{k}+}\right)$ or NOD/SCID $\left(\mathrm{H}-2 \mathrm{~K}^{\mathrm{k}-}\right)$ mice. Percent $\mathrm{H}-2 \mathrm{~K}^{\mathrm{k}+}$ cells in the blood was determined by flow cytometry at 1 and 2 months following transplantation. Results (mean \pm SEM) are based on 15 NOD/SCID mice per group. 